\title{
CME Cerebrovascular disease (108460): self-assessment questionnaire
}

\author{
Authors: John Bamford and Tahseen A Chowdhury
}

SAQs and answers are ONLINE for RCP fellows and collegiate members

SAQs and answers are ONLINE for RCP fellows and collegiate members

\section{Format}

Candidates are asked to choose the best answer from the five possible answers. This best of five format is used in many medical examinations, however the questions are not intended to be representative of those used in the MRCP(UK) Part 1 or Part 2 Written Examinations.

\section{The answering process}

1 Go to www.rcplondon.ac.uk/SAQ

2 Log on using your usual RCP username and password

3 Select the relevant CME question paper

4 Answer all 10 questions by selecting the best answer from the options provided

5 Once you have answered all the questions, click on Submit

\section{Registering your external CPD credits}

Carrying out this activity allows you to claim two external CPD credits. These will be automatically transferred to your CPD diary, where you can review the activity and claim your points.

1 Which of the following statements about stroke mimics is true?

(a) A high National Institutes of Health Stroke Scale score and the presence of cognitive impairment are predictive of a mimic.

(b) Adverse events from administering thrombolysis to mimics are high.

(c) Patients with migraine have a lower risk of stroke disease than patients without migraine.

(d) Seizure is an absolute contraindication to thrombolysis.

(e) Seizure is the commonest condition mimicking acute stroke, with post-ictal paresis often lasting several hours or even days.

2 Which of the following statements about neuroimaging in acute stroke is correct?

(a) Computerised tomography (CT) angiogram is the first line investigation for all patients with suspected stroke. (b) CT has high sensitivity for acute ischaemia when performed within 6 hours of symptom onset.

(c) Encephalomalacia and hypodensity are signs of early ischaemia on CT.

(d) Restricted diffusion on magnetic resonance imaging can be seen in migraine, sepsis and syncope.

(e) The presence of restricted diffusion on magnetic resonance imaging strongly supports a diagnosis of stroke rather than mimic.

3 With reference to intravenous thrombolysis for acute ischaemic stroke, which of the following statements is true?

(a) Benefit is less likely in those over 80 years old.

(b) Neurological deterioration after intravenous thrombolysis is most commonly due to intracerebral haemorrhage.

(c) Risk of symptomatic intracerebral haemorrhage is higher when treatment is delivered later after symptom onset.

(d) The odds of recovery to independence are greatest when treatment is delivered within 90 minutes of symptom onset.

(e) Treatment is ineffective in those with established abnormalities on brain imaging.

4 In clinical trials of endovascular thrombectomy, which of the following is true?

(a) All trials included patients with angiographically confirmed occlusion of the middle cerebral artery.

(b) Endovascular treatment with any available device has been established to improve outcomes.

(c) Embolisation causing stroke in previously uninvolved vascular territories occurs in more than $25 \%$ of thrombectomy procedures.

(d) Intracerebral haemorrhage was more frequent among patients treated with thrombectomy.

(e) The majority of thrombectomy-treated patients had contraindications to intravenous thrombolysis.

5 Which of the following should be standard components of stroke unit care?

(a) dietetics

(b) occupational therapy

(c) psychology

(d) social services

(e) all of the above. 
6 When a patient is admitted with acute stroke, which out of the following assessments should be completed first?
(a) carotid Doppler
(b) cognitive function
(c) echocardiography
(d) mood
(e) swallow safety.

7 Which of the following best describes the optimal patient care pathway following acute stroke?

(a) admission to a combined stroke unit for as long as is required for the patient to reach their best possible recovery

(b) admission to a general medical ward and then transfer to a rehabilitation ward

(c) admission to a hyperacute stroke unit and then transfer to a care of the elderly ward

(d) admission to a hyperacute stroke unit followed by transfer to a stroke rehabilitation unit linked to an early support discharge team

(e) admission to a hyperacute stroke unit only if the patient is treated with thrombolysis or thrombectomy, then transfer to a stroke rehabilitation unit linked to an early support discharge team.

8 When a patient deteriorates within the first 24 hours of an admission for acute stroke, which of the following is true?

(a) Clinical examination of the patient is always required.

(b) If after thrombolysis, the cause will always be haemorrhage and so urgent brain imaging is not needed.

(c) If the patient requires palliative care then they should be transferred to any medical ward as they no longer require specialist input.

(d) There is not usually much that can be done to improve the patient's outcome.

(e) Urgent computerised tomography brain imaging is always required.

9 A 60 -year-old woman with mechanical mitral valve prosthesis presented with right hemiparesis. She was taking warfarin $5 \mathrm{mg}$ daily with international normalised ratio (INR) of 2.8. Computerised tomography brain showed a left thalamic haematoma. Which of the following is correct?

(a) A novel oral anticoagulant (NOAC) can be restarted 1-2 weeks later when clinically stable, instead of warfarin, as the risk of bleeding is lower with NOACs. (b) Haematology and cardiology opinions should be sought before deciding on the next course of action.

(c) Prothrombin complex concentrates should be given instead of fresh frozen plasma.

(d) The patient should be admitted to the acute cardiac unit for close monitoring because of the risk of valve thrombosis.

(e) Warfarin should be substituted with parental heparin in the acute stage.

10 A 70-year-old man presented with left hemiparesis that started 2 hours previously. His past medical history included hypertension and ischaemic heart disease for which he underwent a coronary artery bypass grafting 10 years earlier. He had an episode of transient ischaemic attack 2 months ago. On examination, his Glasgow Coma Score was 12/15. His blood pressure was $210 / 130 \mathrm{mmHg}$. Computerised tomography brain showed a right basal ganglia haematoma with a volume of $20 \mathrm{~mL}$. Which of the following is correct?

(a) A do-not-resuscitate order should be discussed on admission in view of his intracerebral haemorrhage.

(b) He should be treated with anti-hypertensive agents targeting a conservative level of $180 / 90 \mathrm{mmHg}$ to avoid precipitating an ischaemic event.

(c) No further neuroimaging should be considered, as the cause of the haematoma is most likely hypertension.

(d) Surgical evacuation of clot should be performed.

(e) Treating with anti-hypertensive agents targeting a level of 140/90 $\mathrm{mmHg}$ within 1 hour is safe.

\section{CME Rheumatology SAQ}

Answers to the CME SAQ published in Clinical Medicine in February 2017

$\begin{array}{llllllllll}\text { Q1 } & \text { Q2 } & \text { Q3 } & \text { Q4 } & \text { Q5 } & \text { Q6 } & \text { Q7 } & \text { Q8 } & \text { Q9 } & \text { Q10 } \\ \text { (e) } & \text { (d) } & \text { (c) } & \text { (e) } & \text { (b) } & \text { (b) } & \text { (e) } & \text { (a) } & \text { (e) } & \text { (a) }\end{array}$

